\title{
Advection/diffusion of large scale magnetic field in accretion disks
}

\author{
R. V. E. Lovelace ${ }^{1}$, G. S. Bisnovatyi-Kogan ${ }^{2}$, and D. M. Rothstein ${ }^{3}$ \\ ${ }^{1}$ Department of Astronomy, Cornell University, Ithaca, NY 14853, USA \\ ${ }^{2}$ Space Research Institute, Russian Academy of Sciences, Moscow, Russia \\ ${ }^{3}$ Department of Astronomy, Cornell University, Ithaca, NY 14853, USA
}

Received: 30 October 2008 - Revised: 12 January 2009 - Accepted: 12 January 2009 - Published: 13 February 2009

\begin{abstract}
Activity of the nuclei of galaxies and stellar mass systems involving disk accretion to black holes is thought to be due to (1) a small-scale turbulent magnetic field in the disk (due to the magneto-rotational instability or MRI) which gives a large viscosity enhancing accretion, and (2) a large-scale magnetic field which gives rise to matter outflows and/or electromagnetic jets from the disk which also enhances accretion. An important problem with this picture is that the enhanced viscosity is accompanied by an enhanced magnetic diffusivity which acts to prevent the build up of a significant large-scale field. Recent work has pointed out that the disk's surface layers are non-turbulent and thus highly conducting (or non-diffusive) because the MRI is suppressed high in the disk where the magnetic and radiation pressures are larger than the thermal pressure. Here, we calculate the vertical ( $z$ ) profiles of the stationary accretion flows (with radial and azimuthal components), and the profiles of the largescale, magnetic field taking into account the turbulent viscosity and diffusivity due to the MRI and the fact that the turbulence vanishes at the surface of the disk. We derive a sixth-order differential equation for the radial flow velocity $v_{r}(z)$ which depends mainly on the midplane thermal to magnetic pressure ratio $\beta>1$ and the Prandtl number of the turbulence $\mathcal{P}=$ viscosity/diffusivity. Boundary conditions at the disk surface take into account a possible magnetic wind or jet and allow for a surface current in the highly conducting surface layer. The stationary solutions we find indicate that a weak $(\beta>1)$ large-scale field does not diffuse away as suggested by earlier work.
\end{abstract}

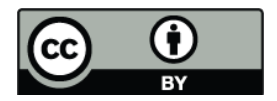

Correspondence to: R. V. E. Lovelace (rvl1@ cornell.edu)

\section{Introduction}

Early work on disk accretion to a black hole argued that a large-scale poloidal magnetic field originating from say the interstellar medium, would be dragged inward and greatly compressed near the black hole by the accreting plasma (Bisnovatyi-Kogan and Ruzmaikin, 1974, 1976) and that this would be important for the formation of jets (Lovelace, 1976). Later, the importance of a weak small-scale magnetic field within the disk was recognized as the source of the turbulent viscosity of disk owing to the magneto-rotational instability (MRI; Balbus and Hawley, 1991). Analysis of the diffusion and advection of a large-scale field in a disk with a turbulent viscosity comparable to the turbulent magnetic diffusivity (as suggested by MRI simulations) indicated that a weak large-scale field would diffuse outward rapidly (van Ballegooijen, 1989; Lubow, Papaloizou, and Pringle, 1994; Lovelace, Romanova, and Newman, 1994, 1997). This has led to the suggestion that special conditions (nonaxisymmetry) are required for the field to be advected inward (Spruit and Uzdensky, 2005). Recently, Bisnovatyi-Kogan and Lovelace (2007) pointed out that the disk's surface layers are highly conducting (or non-diffusive) because the MRI is suppressed in this region where the magnetic energy-density is larger than the thermal energy-density. Rothstein and Lovelace (2008) analyzed this problem in further detail and discussed the connections with global and shearing box magnetohydrodynamic (MHD) simulations of the MRI.

Here we calculate the profiles through the disk of stationary accretion flows (with radial and azimuthal components), and the profiles of a large-scale, weak magnetic field taking into account the turbulent viscosity and diffusivity due to the MRI (in an $\alpha$-description) and the fact that the turbulence vanishes at the surface of the disk. A full explanation of this work will be given elsewhere (Lovelace, Rothstein, and Bisnovatyi-Kogan, 2009). Related calculations of the

Published by Copernicus Publications on behalf of the European Geosciences Union and the American Geophysical Union. 
disk structure were done earlier by Königl (1989), Li (1995), Ogilvie and Livio (2001) but without taking into account the absence of turbulence at the disk's surface. Recent work calls into question the $\alpha$-description of the MRI turbulence in accretion disks and develops a closure model which fits shearing box simulation results (Pessah, Chan, and Psaltis, 2008). Furthermore, there is evidence that the MRI turbulence retains dependences on the microscopic viscosity and diffusivity and consequently on the microscopic Prandtl number (Lesur et al., 2007; Fromang et al., 2007). Analysis of these issues is deferred to a future study.

\section{Theory}

We consider the non-ideal magnetohydrodynamics of a thin axisymmetric, viscous, resistive disk threaded by a largescale dipole-symmetry magnetic field $\mathbf{B}$. We use a cylindrical $(r, \phi, z)$ inertial coordinate system in which the timeaveraged magnetic field is $\mathbf{B}=B_{r} \hat{\mathbf{r}}+B_{\phi} \hat{\boldsymbol{\phi}}+B_{z} \hat{\mathbf{z}}$, and the timeaveraged flow velocity is $\mathbf{v}=v_{r} \hat{\mathbf{r}}+v_{\phi} \hat{\boldsymbol{\phi}}+v_{z} \hat{\mathbf{z}}$. The main equations are

$\rho \frac{d \mathbf{v}}{d t}=-\nabla p+\rho \mathbf{g}+\frac{1}{c} \mathbf{J} \times \mathbf{B}+\mathbf{F}^{v}$,

$\frac{\partial \mathbf{B}}{\partial t}=\nabla \times(\mathbf{v} \times \mathbf{B})-\nabla \times(\eta \nabla \times \mathbf{B})$.

These equations are supplemented by the continuity equation, by $\nabla \times \mathbf{B}=4 \pi \mathbf{J} / c$, and by $\nabla \cdot \mathbf{B}=0$. Here, $\eta$ is the magnetic diffusivity, $\mathbf{F}^{v}=-\nabla \cdot T^{v}$ is the viscous force with $T_{j k}^{v}=-\rho \nu\left(\partial v_{j} / \partial x_{k}+\partial v_{k} / \partial x_{j}-(2 / 3) \delta_{j k} \nabla \cdot \mathbf{v}\right)$ (in Cartesian coordinates), and $v$ is the kinematic viscosity.

We assume that both the viscosity and the diffusivity are due to magneto-rotational (MRI) turbulence in the disk so that

$\nu=\mathcal{P} \eta=\alpha \frac{c_{s 0}^{2}}{\Omega_{K}} g(z)$,

where $\mathcal{P}$ is the magnetic Prandtl number of the turbulence assumed a constant of order unity, $\alpha \leq 1$ is the dimensionless Shakura-Sunyaev (1973) parameter, $c_{S}$ is the midplane isothermal sound speed, $\Omega_{K} \equiv\left(G M / r^{3}\right)^{1 / 2}$ is the Keplerian angular velocity of the disk, and $M$ is the mass of the central object. The function $g(z)$ accounts for the absence of turbulence in the surface layer of the disk (Bisnovatyi-Kogan and Lovelace, 2007; Rothstein and Lovelace, 2008). In the body of the disk $g=1$, whereas at the surface of the disk, at say $z_{S}$, $g$ tends over a short distance to a very small value $\sim 10^{-8}$, effectively zero, which is the ratio of the Spitzer diffusivity of the disk's surface layer to the turbulent diffusivity of the body of the disk.

We consider stationary solutions of Eqs. (1) and (2) for a weak large-scale magnetic field. These can be greatly simplified for thin disks where the disk half-thickness, of the order of $h \equiv c_{s 0} / \Omega_{K}$, is much less than $r$. Thus we have the small parameter $\varepsilon=h / r=c_{s 0} / v_{K} \ll 1$. In the following we use the dimensionless height $\zeta \equiv z / h$. The three magnetic field components are assumed to be of comparable magnitude on the disk's surface, but $B_{r}=0=B_{\phi}$ on the midplane. On the other hand the axial magnetic field changes by only a small amount going from the midplane to the surface, $\Delta B_{z} \sim \varepsilon B_{r} \ll B_{z}$ (from $\nabla \cdot \mathbf{B}=0$ ) so that $B_{z} \approx$ const inside the disk. As a consequence, the $\partial B_{j} / \partial r$ terms in the magnetic force in Eq. (1) can all be dropped in favor of the $\partial B_{j} / \partial z$ terms (with $j=r, \phi$ ). The velocity components are assumed to satisfy $v_{z}^{2} \ll c_{s 0}^{2}$ and $v_{r}^{2} \ll v_{\phi}^{2}$. Consequently, $v_{\phi}(r, z)$ is close in value to the Keplerian value $v_{K}(r) \equiv(G M / r)^{1 / 2}$. Thus, $\partial v_{\phi} / \partial r=-(1 / 2)\left(v_{\phi} / r\right)$ to a good approximation.

With these assumptions, the radial component of Eq. (1) gives

$\frac{\partial b_{r}}{\partial \zeta}=\frac{\beta \tilde{\rho}}{\varepsilon}\left(1-k_{p} \varepsilon^{2}-u_{\phi}^{2}\right)+\alpha^{2} \beta \frac{\partial}{\partial \zeta}\left(\tilde{\rho} g \frac{\partial u_{r}}{\partial \zeta}\right)$,

where $\tilde{\rho} \equiv \rho(r, z) / \rho_{0}$ with $\rho_{0} \equiv \rho(r, z=0)$. The midplane plasma beta is $\beta \equiv 4 \pi \rho_{0} c_{s 0}^{2} / B_{0}^{2}$, where $k_{p} \equiv-\partial \ln p / \partial \ln r$ is assumed of order unity and $p=\rho c_{s}^{2}$. Note that $\beta=c_{s 0}^{2} / v_{A 0}^{2}$, where $v_{A 0}=B_{0} /\left(4 \pi \rho_{0}\right)^{1 / 2}$ is the midplane Alfvén velocity. The rough condition for the MRI instability and the associated turbulence in the disk is $\beta>1$ (Balbus and Hawley, 1991). In the following we assume $\beta>1$, which we refer to as a weak magnetic field. We normalize the field components by $B_{0}=B_{z}(r, z=0)$, with $b_{r}=B_{r} / B_{0}, b_{\phi}=B_{\phi} / B_{0}$, and $b_{z}=B_{z} / B_{0} \approx 1$. Also, $u_{\phi} \equiv v_{\phi}(r, z) / v_{K}(r)$ and the accretion speed $u_{r} \equiv-v_{r} /\left(\alpha c_{s 0}\right)$. For the assumed dipole field symmetry, $b_{r}$ and $b_{\phi}$ are odd functions of $\zeta$ whereas $u_{r}$ and $u_{\phi}$ are even functions.

In a similar way one can derive an equation for $\partial b_{\phi} / \partial \zeta$ from the toroidal component of Eq. (1). The $z$-component of Eq. (1) corresponds to hydrostatic equilibrium for $\beta>1$. Equations for $\partial u_{\phi} / \partial \zeta$ and $\partial b_{r} / \partial \zeta$ follow from Eq. (2).

Integration of the $\partial b_{\phi} / \partial \zeta$ equation from $\zeta=0$ (where $b_{\phi}=0$ and $\left.\partial u_{\phi} / \partial \zeta=0\right)$ to the exterior of the disk $\left(\zeta_{S+}\right.$ where $g=0)$ gives the average accretion speed,

$\bar{u}_{r}=u_{0}-\frac{2 b_{\phi S+}}{\alpha \beta \tilde{\Sigma}}$,

which is the sum of a viscous contribution, $u_{0} \equiv 3 \varepsilon k_{v}$ (with $k_{\nu} \equiv \partial \ln \left(\rho c_{s 0}^{2} r^{2} / h\right) / \partial \ln (r)>0$ of order unity), and a magnetic contribution $\left(\propto b_{\phi S+}\right)$ due to the loss of angular momentum from the surface of the disk where necessarily $b_{\phi S+} \leq 0$ (Lovelace, Romanova, and Newman, 1994). Here $\bar{u}_{r} \equiv \int_{0}^{\zeta_{S}} d \zeta \tilde{\rho} u_{r} / \tilde{\Sigma}, \tilde{\Sigma} \equiv \int_{0}^{\zeta_{S}} d \zeta \tilde{\rho}$, and the $S+$ subscript indicates evaluation outside the disk. A similar integration of the $\partial b_{r} / \partial \zeta$ equation implies that

$b_{r S}=\mathcal{P} \zeta_{S}\left\langle u_{r}\right\rangle$

where $\langle\ldots\rangle=\int_{0}^{\zeta S} d \zeta(\ldots) / \zeta_{S}$. 
The equations for $u_{r}, u_{\phi}, b_{r}, \& b_{\phi}$ can be combined to give a single equation for $u_{r}(\zeta)$,

$$
\begin{array}{r}
\alpha^{4} \beta^{2} \frac{\partial^{2}}{\partial \zeta^{2}}\left(g \frac{\partial}{\partial \zeta}\left(\tilde{\rho} g \frac{\partial}{\partial \zeta}\left(\frac{1}{\tilde{\rho}} \frac{\partial}{\partial \zeta}\left(\tilde{\rho} g \frac{\partial u_{r}}{\partial \zeta}\right)\right)\right)\right) \\
-\alpha^{2} \beta \mathcal{P} \frac{\partial^{2}}{\partial \zeta^{2}}\left(g \frac{\partial}{\partial \zeta}\left(\tilde{\rho} g \frac{\partial}{\partial \zeta}\left(\frac{u_{r}}{\tilde{\rho} g}\right)\right)\right) \\
-\alpha^{2} \beta \mathcal{P} \frac{\partial^{2}}{\partial \zeta^{2}}\left(\frac{1}{\tilde{\rho}} \frac{\partial}{\partial \zeta}\left(\tilde{\rho} g \frac{\partial u_{r}}{\partial \zeta}\right)\right) \\
+\alpha^{2} \beta^{2} \frac{\partial^{2}}{\partial \zeta^{2}}\left(\tilde{\rho} g\left(u_{r}-g u_{0}\right)\right)+\mathcal{P}^{2} \frac{\partial^{2}}{\partial \zeta^{2}}\left(\frac{u_{r}}{\tilde{\rho} g}\right) \\
+3 \beta \mathcal{P}^{2} \frac{u_{r}}{g}=0 .
\end{array}
$$

This equation is integrated from $\zeta=0$ out to the surface of the disk $\zeta_{S}$ where the boundary conditions apply. Because $u_{r}$ is an even function of $\zeta$, only $u_{r}(0), u_{r}^{\prime \prime}(0)$, and $u_{r}^{i v}(0)$ need to be adjusted in order to satisfy the boundary conditions at the disk surface (Lovelace et al., 2009).

Note that the value of $b_{\phi S+} \leq 0$ is not fixed by the solution for the field/flow inside the disk. Its value can be determined by matching the calculated surface fields $b_{r}$ and $b_{\phi S+}$ onto an external magnetic wind or jet solution. Stability of the wind or jet solution to current driven kinking is predicted to limit the ratio of the toroidal to axial magnetic field components at the disk's surface $\left|b_{\phi S+}\right|$ to values $\lesssim \mathcal{O}\left(2 \pi r / L_{z}\right)$ (Hsu and Bellan, 2002; Nakamura, Li, and Li, 2007), where $L_{z}$ is the length-scale of field divergence of the wind or jet at the disk surface. From known wind and jet solutions we estimate $2 \pi r / L_{z} \approx \pi$ (Lovelace, Berk, and Contopoulos, 1991; Ustyugova et al., 1999; Ustyugova et al., 2000; Lovelace et al., 2002). The quantity $\bar{u}_{r} / u_{0}-1=2\left|b_{\phi S+}\right| /\left(\alpha \beta \tilde{\Sigma} u_{0}\right)$ is the faction of the accretion power going into the jets or winds (Lovelace et al., 1994). For the mentioned upper limit on $\left|b_{\phi S+}\right|$, we find $\bar{u}_{r} / u_{0}-1 \lesssim \mathcal{O}\left[2 \pi /\left(\alpha \beta \tilde{\Sigma} u_{0}\right)\right]$. From Eq. (6) we have $b_{r S}=\left(\mathcal{P} \zeta_{S} u_{0}\right)\left(\left\langle u_{r}\right\rangle / u_{0}\right)$. Therefore, for $\beta \gg 1$ and $\left\langle u_{r}\right\rangle \approx u_{0}$, we have $b_{r S} \approx \mathcal{P} \zeta S u_{0}$.

The matching of internal and external field/flow solutions has been carried out by Königl (1989) and Li (1995) for the case of self-similar $\left[B_{z}(r, 0) \sim r^{-5 / 4}\right]$ magnetocentrifugally outflows from the disk's surface. These outflows occur under conditions where the poloidal field lines at the disk's surface are tipped relative to the rotation axis by more than $30^{\circ}$ which corresponds to $b_{r} S>3^{-1 / 2} \approx 0.577$ (Blandford and Payne, 1982). The outflows typically carry a significant mass flux. For the internal field/flow solutions discussed in $\S 4$ with $\beta \gg 1$, we conclude that $b_{r S}$ is sufficiently large for magnetocentrifugal outflows only for turbulent magnetic Prandtl numbers, $\mathcal{P} \gtrsim 2.7$. Shu and collaborators (e.g., Cai et al., 2008, and references therein) have developed detailed "Xwind" models which depend on the disk having Prandtl numbers larger than unity.

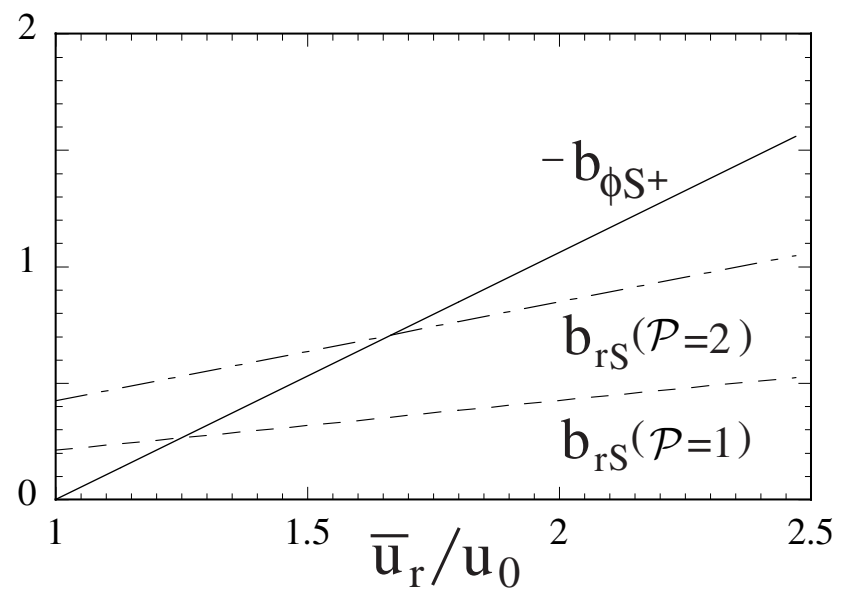

Fig. 1. Radial and toroidal field components (normalized to $B_{z}$ ) at the disk's surface as a function of the average accretion speed $\bar{u}_{r}$ (normalized by the viscous accretion speed $u_{0}$ ). For this plot $\beta=100$ and Prandtl numbers $\mathcal{P}=1$ and 2 . Note that $b_{\phi S+}$ is given by Eq. (5) and is independent of $\mathcal{P}$ and $b_{r S}$ is given by Eq. (6).

For Prandtl numbers say $\mathcal{P} \lesssim 2.7$, the values of $b_{r S}$ are too small for there to be a magnetocentrifugal outflow. In this case there is an outflow of electromagnetic energy and angular momentum from the disk (with little mass outflow) in the form of a magnetically dominated or "Poynting flux jet" (Lovelace, Wang, and Sulkanen, 1987; Lovelace, et al., 2002) also referred to as a "magnetic tower jet" (LyndenBell, 1996, 2003). MHD simulations have established the occurrence of Poynting-flux jets under different conditions (Ustyugova et al., 2000, 2006; Kato, Kudoh, and Shibata, 2002; Kato, 2007). Laboratory experiments have allowed the generation of magnetically dominated jets (Hsu and Bellan, 2002; Lebedev et al., 2005).

Figure 1 shows the dependences of the surface field components on the accretions speed for $\alpha=0.1$ and $\beta=100$.

Figures 2 and 3 show a sample solution of Eq. (7) for $\varepsilon=0.05, \alpha=0.1, \beta=100$, and $\mathcal{P}=1$ where we find $\bar{u}_{r} / u_{0}=1.30, b_{\phi S+}=-0.321$, and $b_{r S}=0.276$. For this solution both the density $\rho(\zeta)$ and $g(\zeta)$ are take to be step functions going to zero at $\zeta_{m}=\sqrt{2}$ (see Lovelace et al., 2009). Note that the solution has a "channel" structure with the midplane region of the disk flowing radially outward and the regions closer to the disk's surfaces flowing radially inward.

\section{Conclusions}

A study is made of stationary axisymmetric accretion flows $\left[v_{r}(z), v_{\phi}(z), v_{z} \approx 0\right]$ and the large-scale, weak magnetic field $\left[B_{r}(z), B_{\phi}(z), B_{z} \approx\right.$ const] taking into account the turbulent viscosity and diffusivity due to the MRI and the fact that the turbulence vanishes at the surface of the disk as discussed by Bisnovayi-Kogan and Lovelace (2007) and Rothestein and 


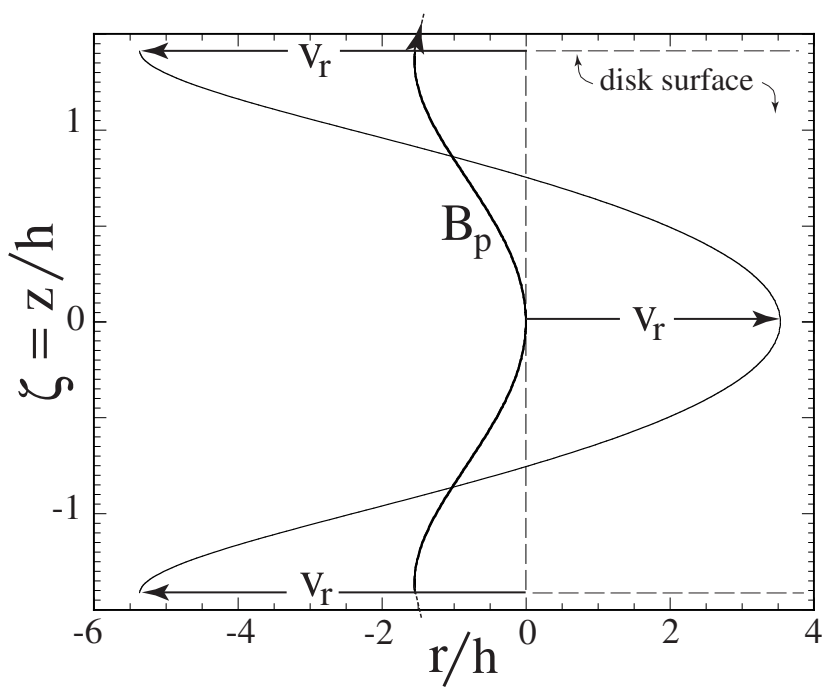

Fig. 2. Radial flow speed $v_{r}=-u_{r}$ (normalized to $\alpha c_{s 0}$ ) as a function of $\zeta=z / h$ and a sample poloidal $\left(B_{r}, B_{z}\right)$ magnetic field line for $\beta=10^{2}$ and $\mathcal{P}=1$.

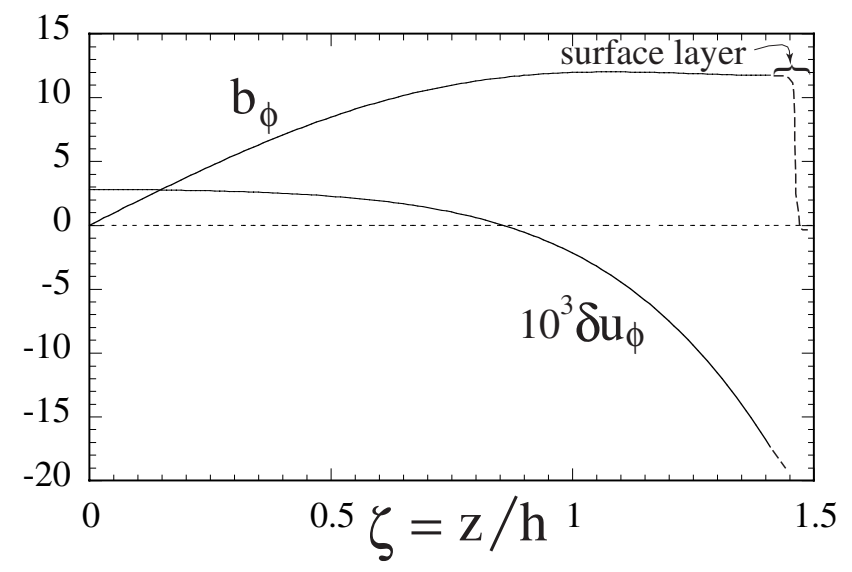

Fig. 3. Toroidal magnetic field $b_{\phi}=B_{\phi} / B_{z}$ and toroidal velocity $\delta u_{\phi}=\left(v_{\phi}-v_{K}\right) / v_{K}$ (with $v_{K}$ the Keplerian velocity) for the case where $\beta=100$ and $\mathcal{P}=1$. The jump in the toroidal magnetic field at the disk's surface is shown by the dashed line.

Lovelace (2008). We derive a sixth-order differential equation for the radial flow velocity $v_{r}(z)$ which depends mainly on the ratio of the midplane thermal to magnetic pressures $\beta>1$ and the Prandtl number of the turbulence $\mathcal{P}=$ viscosity/diffusivity. Boundary conditions at the disk's surfaces take into account the outflow of angular momentum to magnetic winds or jets and allow for a surface current flow in the highly conducting surface layers. In general we find that there is a radial surface current but no toroidal surface current. Stability of the winds or jets will limit the ratio of the toroidal to axial field at the disk's surface $\left|b_{\phi S+}\right|$ to values $\lesssim \pi$. The stationary solutions we find indicate that a weak $(\beta \gg 1)$, large-scale field does not diffuse away as suggested by earlier work (e.g., Lubow et al., 1994) which assumed $b_{r S} \geq 3^{-1 / 2}$.

The flow/field solutions found here in a viscous/diffusive disk and are different from the exponentially growing channel flow solutions found by Goodman and Xu (1994) for an MRI in an ideal MHD unstable shearing box. Channel solutions in viscous/diffusive disks were found earlier by Ogilvie and Livio (2001) and by Salmeron, Königl, and Wardle (2007) for conditions different from those considered here. In general we find that the magnitude of the toroidal magnetic field component inside the disk is much larger than the other field components. The fact that the viscous accretion speed is very small, $\sim \alpha \varepsilon c_{s 0}$, means that even a small large-scale field can significantly influence the accretion flow. We find that Prandtl numbers larger than a critical value estimated to be 2.7 are needed in order for there to be magnetocentrifugal outflows from the disk's surface. For smaller $\mathcal{P}$, electromagnetic outflows are predicted. Owing to the stability condition, $\left|b_{\phi S+}\right| \lesssim \pi$, the fraction of the accretion power going into magnetic outflows or jets is $\lesssim$ const $\beta^{-1} \sim B_{z}^{2}$.

Analysis of the time-dependent accretion of the large-scale B-field is clearly needed to study the amplification of the field and build up of magnetic flux in the inner region of the disk. One method is to use global 3-D MHD simulations (Igumenshchev et al., 2003; Hirose et al., 2004; De Villiers et al., 2005; Hawley and Krolik, 2006; McKinney and Narayan, 2007; Igumenshchev, 2008), but this has the difficulty of resolving the very thin highly conducting surface layers of the disk. Another method is to generalize the approach of Lovelace et al. (1994) taking into account the results of the present work. This is possible because the radial accretion time $\left(r /\left|\bar{u}_{r}\right|\right)$ is typically much longer than the viscous diffusion time across the disk $\left(h^{2} / v\right)$.

Acknowledgement. R. V. E. L. was supported in part by NASA grant NNX08AH25G and by NSF grants AST-0607135 and AST-0807129. G. B.-K. was partially supported by RBFR grants 08-02-00491 and 08-02-90106, and RAN program P-04. D. M. R. is supported by an NSF Astronomy and Astrophysics Postdoctoral Fellowship under award AST-0602259.

Edited by: T. Passot

Reviewed by: P. Hennebelle and another anonymous referee

\section{References}

Balbus, S. A. and Hawley, J. F.: A powerful local shear instability in weakly magnetized disks. I. linear analysis, Astrophys. J., 376, 214-222, 1991.

Bisnovatyi-Kogan, G. S. and Ruzmaikin, A. A.: The accretion of matter by a collapsing star in the presence of a magnetic field, Astrophys. Space Sci., 28, 45-59, 1974.

Bisnovatyi-Kogan, G. S. and Ruzmaikin, A. A.: The accretion of matter by a collapsing star in the presence of a magnetic field, 
II. Self-consistent stationary picture, Astrophys. Space Sci., 42, 401-424, 1976.

Bisnovatyi-Kogan, G. S. and Lovelace, R. V. E.: Large-scale B-field in stationary accretion disks, Astrophys. J., 667, L167-L169, 2007.

Blandford, R. D. and Payne, D. G.: Hydromagnetic flows from accretion discs and the production of radio jets, Mon. Not. R. Astron. Soc., 199, 883-903, 1982.

Cai, M. J., Shang, H., Lin, H. H., and Shu, F. H.: X-winds in action, Astrophys. J., 672, 489-503, 2008.

De Villiers, J.-P., Hawley, J. F., Krolik, J. H., and Hirose, S.: Magnetically driven accretion in the Kerr metric, III. Unbound outflows, Astrophys. J., 620, 878-888, 2005.

Fromang, S., Papaloizou, J., Lesur, G., and Heinemann, T.: MHD simulations of the magnetorotational instability in a shearing box with zero net flux, II. The effect of transport coefficients, Astron. Astrophys., 476, 1123-1132, 2007.

Goodman, J. and Xu, G.: Parasitic instabilities in magnetized differentially rotating disks, Astrophys. J., 432, 213-223, 1994.

Hawley, J. F. and Krolik, J. H.: Magnetically driven jets in the Kerr metric, Astrophys. J., 641, 103-116, 2006.

Hirose, S., Krolik, J. H., De Villiers, J.-P., and Hawley, J. F.: Magnetically Driven Accretion Flows in the Kerr metric, II. Structure of the magnetic field, Astrophys. J., 606, 1083-1097, 2004.

Hsu, S. C. and Bellan, P. M.: A laboratory plasma experiment for studying magnetic dynamics of accretion disks and jets, Mon. Not. R. Astron. Soc., 334, 257-261, 2002.

Igumenshchev, I. V., Narayan, R., and Abramowicz, M. A.: Threedimensional magnetohydrodynamic simulations of radiatively inefficient accretion flows, Astrophys. J., 592, 1042-1059, 2003.

Igumenshchev, I. V.: Magnetically arrested disks and the origin of Poynting jets: a numerical study, Astrophys. J., 677, 317-326, 2008.

Kato, S. X., Kudoh, T., and Shibata, K.: 2.5 dimensional non steady magnetohydrodynamic simulations of magnetically driven jets from geometrically thin disks, Astrophys. J., 565, 1035-1049, 2002.

Kato, Y.: Magnetic tower jet solution for launching astrophysical jets, A\&SS, 307, 11-15, 2007.

Königl, A.: Self-similar models of magnetized accretion disks, Astrophys. J., 342, 208-223, 1989

Lebedev, S. V., Ciardi, A., Ampleford, D. J., Bland, S. N., Bott, S. C., Chittenden, J. P., Hall, G. N., Rapley, J., Jennings, C. A., Frank, A., Blackman, E. G., and Lery, T.: Magnetic tower outflows from a radial wire array Z-pinch, Mon. Not. R. Astron. Soc., 361, 97-108, 2005.

Lesur, G. and Longaretti, P.-Y.: Impact of dimensionless numbers on the efficiency of magnetorotational instability induced turbulent transport, Mon. Not. R. Astron. Soc., 378, 1471-1480, 2007.

Li, Z.-Y.: Magnetohydrodynamic disk-wind connection: selfsimilar solutions, Astrophys. J., 444, 848-860, 1995.

Lovelace, R. V. E.: Dynamo model of double radio sources, Nature, 262, 649-652, 1976.

Lovelace, R. V. E., Wang, J. C. L., and Sulkanen, M. E.: Self- collimated electromagnetic jets from magnetized accretion disks, Astrophys. J., 315, 504-535, 1987.
Lovelace, R. V. E., Berk, H. L., and Contopoulos, J.: Magnetically driven jets and winds, Astrophys. J., 379, 696-705, 1991.

Lovelace, R. V. E., Romanova, M. M., and Newman, W. I.: Implosive accretion and outbursts of active galactic nuclei, Astrophys. J., 437, 136-143, 1994.

Lovelace, R. V. E., Newman, W. I., and Romanova, M. M.: Jet outbursts from fast accretion in a disk with zebra-stripe magnetic field, Astrophys. J., 484, 628-636, 1997.

Lovelace, R. V. E., Rothstein, D. M., and Bisnovatyi-Kogan, G. S.: Advection/Diffusion of large-scale B-field in accretion disks, in preparation, 2009.

Lubow, S. H., Papaloizou, J. C. B., and Pringle, J. E.: Magnetic field dragging in accretion discs, Mon. Not. R. Astron. Soc., 267, 235-240, 1994.

Lynden-Bell, D.: Magnetic collimation by accretion discs of quasars and stars, Mon. Not. R. Astron. Soc., 279, 389-401, 1996.

Lynden-Bell, D.: On why discs generate magnetic towers and collimate jets, Mon. Not. R. Astron. Soc., 341, 1360-1372, 2003.

McKinney, J. C. and Narayan, R.: Disc-jet coupling in black hole accretion systems - I. General relativistic magnetohydrodynamic models, Mon. Not. R. Astron. Soc., 375, 513-530, 2007.

Nakamura, M., Li, H., and Li, S.: Stability properties of magnetic tower jets, Astrophys. J., 656, 721-732, 2007.

Ogilvie, G. I. and Livio, M.: Launching of jets and the vertical structure of accretion disks, Astrophys. J., 553, 158-173, 2001.

Pessah, M. E., Chan, C.-k, and Psaltis, D.: The fundamental difference between shear alpha viscosity and turbulent maagnetorotational stresses, Mon. Not. R. Astron. Soc., 383, 683-690, 2007.

Rothstein, D. M. and Lovelace, R. V. E.: Advection of magnetic fields in accretion disks: Not so difficult after all, Astrophys. J., 677, 1221-1232, 2008.

Salmeron, R., Königl, and Wardle, M.: Angular momentum transport in protostellar discs, Mon. Not. R. Astron. Soc., 375, 177183, 2007.

Shakura, N. I. and Sunyaev, R. A.: Black holes in binary systems: Observational appearance, Astron. Astrophys., 24, 337355, 1973.

Spruit, H. C. and Uzdensky, D. A.: Magnetic flux captured by an accretion disk, Astrophys. J., 629, 960-968, 2005.

Ustyugova, G. V., Koldoba, A. V., Romanova, M. M., Chechetkin, V. M., and Lovelace, R. V. E.: Magnetocentrifugally driven winds: Comparison of MHD simulations with theory, Astrophys. J., 516, 221-235, 1999.

Ustyugova, G. V., Lovelace, R. V. E., Romanova, M. M., Li, H., and Colgate, S. A.: Poynting jets from accretion disks, Magnetohydrodynamic simulations, Astrophys. J., 541, L21-L24, 2000.

Ustyugova, G. V., Koldoba, A. V., Romanova, M. M., and Lovelace, R. V. E.: Propeller regime of disk accretion to rapidly rotating stars, Astrophys. J., 646, 304-318, 2006.

van Ballegooijen, A. A. : Magnetic fields in the accretion disks of cataclysmic variables, in: Accretion disks and magnetic fields in astrophysics: Proceedings of the European Physical Society study conference held in Noto (Sicily), Italy, 16-21 June 1988, edited by: Belvedere, G., 99-106, (Dordrecht; Boston: Kluwer Academic Publishers), 1989. 